\title{
REAL-TIME ROAD TRAFFIC MONITORING USING A FAST A PRIORI KNOWLEDGE BASED SAR-GMTI ALGORITHM
}

\author{
Stefan V. Baumgartner, Gerhard Krieger \\ Microwaves and Radar Institute, German Aerospace Center (DLR) \\ Muenchner Strasse 20, 82234 Wessling, GERMANY, Email: stefan.baumgartner@dlr.de
}

\begin{abstract}
Radar systems operating on high altitude platforms can provide traffic information over wide areas, independent of sunlight illumination and weather conditions. In the paper, a novel a priori knowledge based ground moving target indication (GMTI) and parameter estimation algorithm applicable on single- as well as on multi-channel synthetic aperture radar (SAR) data is presented. Only the intersection points of the moving vehicle signals with the a priori known road axes, which are mapped into the range-compressed data domain, are evaluated. The algorithm needs low computational load and is hence well suited for real-time traffic monitoring applications.
\end{abstract}

Index Terms - Synthetic aperture radar, pulse Doppler radar, radar signal processing, road vehicle location

\section{INTRODUCTION}

Nowadays, a lot of motorways are equipped with sensors to monitor the actual traffic situation with the aim to ensure mobility (avoid congestions) and to increase the safety of road users. Unfortunately, such detailed traffic information is missing outside the major motorways due to the lack of sensor installations. Radars flying at high altitudes provide an elegant solution to fill this gap, especially if this information is required only on a non-regular basis as in the case of special events or catastrophes. For this, a new radar based traffic monitoring system is currently being developed by the Microwaves and Radar Institute of the German Aerospace Center (DLR). This airborne system has the challenging task to acquire, process and deliver the relevant traffic products to a dedicated traffic management center in real-time. SAR and GMTI processing have to be carried out directly onboard the aircraft (cf. Fig. 1). Due to bandwidth limitations, only the relevant traffic data are transmitted to a ground station using a laser communication terminal or a microwave downlink. After further processing the data are forwarded to the traffic management center.

Principally already existing GMTI systems and algorithms originated in the military field can be used for moving vehicle detection and parameter estimation. However, most

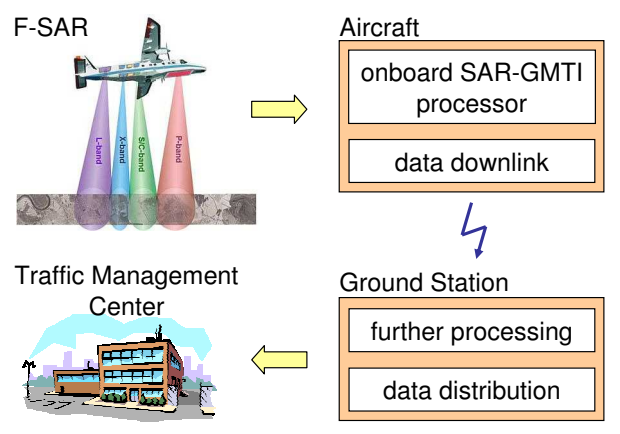

Fig. 1. Radar based traffic monitoring concept.

of these algorithms require large computing power and, if the computation should be performed in real time, the system complexity and the costs become astronomical. For traffic monitoring applications each vehicle has to be assigned to a certain road. For this task anyway a road data base is required. Furthermore, it is not necessary to detect vehicles moving off-road. Hence, by incorporating the a priori known road network already into the detection stage of the GMTI algorithm and by ignoring off-road moving vehicles, the system complexity, the costs as well as the computational load can be reduced significantly.

The idea to use a road network is not new, but up to now the road network mainly was used together with displacement based GMTI algorithms. These algorithms measure the azimuth displacements of the vehicles, occurred due to conventional SAR focusing, for computing the across-track velocities [1]. The required processing is time consuming since in general SAR images have to be generated taking into account the full bandwidth given by the pulse repetition frequency.

Our proposed algorithm does not require SAR focusing, since it operates on single- or multi-channel rangecompressed SAR data. The geocoded position of each detected moving vehicle is directly obtained from the intersection of the road axis with the range-compressed moving vehicle signal. Motion parameter computation is done by estimating the Doppler frequency of the signal at the road intersection. The parameters absolute velocity, heading and geocoded position can be estimated with high accuracy. 


\section{ALGORITHM}

\subsection{Principle}

As a first step the a priori known road axis is mapped into the range-compressed SAR data array. The required coordinate transformation, which is the heart of the proposed algorithm, is done such that the geographical coordinates of each road point are transformed to corresponding beam center coordinates in the range/azimuth plane. The beam center position of a detected moving vehicle is then given by the intersection of the vehicle signal with the mapped road point (cf. Fig. 2).
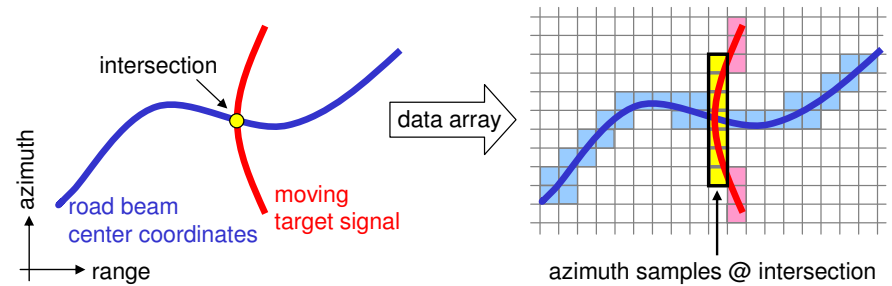

Fig. 2. Principle of the proposed algorithm.

Owing to the mapping the geographical coordinates of the road point and, hence, the coordinates of the detected vehicle moving on this road point at beam center time $t_{b c}$ are known, so that no further geocoding is required. For moving vehicle detection and motion parameter estimation only a few azimuth samples around the intersection point are taken (cf. Fig. 2 right) and transformed into Doppler domain via FFT. Due to the small number of used azimuth samples, the signal phase is more or less linear over time and so the moving vehicle signal appears as a sharp peak in Doppler domain. For detection the signal amplitude is compared to a certain threshold and for motion parameter estimation the Doppler shift $f_{D C}$ of the signal peak is exploited. The proposed algorithm is well suited for airborne but not for spaceborne applications, since the detection performance suffers from low SNR.

\subsection{Structure of the Algorithm}

In Fig. 3 the flow chart of the proposed GMTI algorithm is shown exemplarily for a dual-channel system. RX1 and $\mathrm{RX} 2$ are the range-compressed images. Clutter suppression is performed using the displaced phase center antenna (DPCA) technique. The geographical coordinates as well as the elevations of the roads of interest are obtained from a road database. Interpolation of these coordinates is necessary to avoid gaps in the range/azimuth plane.

Around each road point in the range/azimuth plane some azimuth samples are extracted from the DPCA data and transformed into Doppler domain using the FFT. Each detected signal peak in the Doppler domain corresponds to a potential moving vehicle. The parameter estimation procedure is explained in section 2.4. Before formatting and distributing the

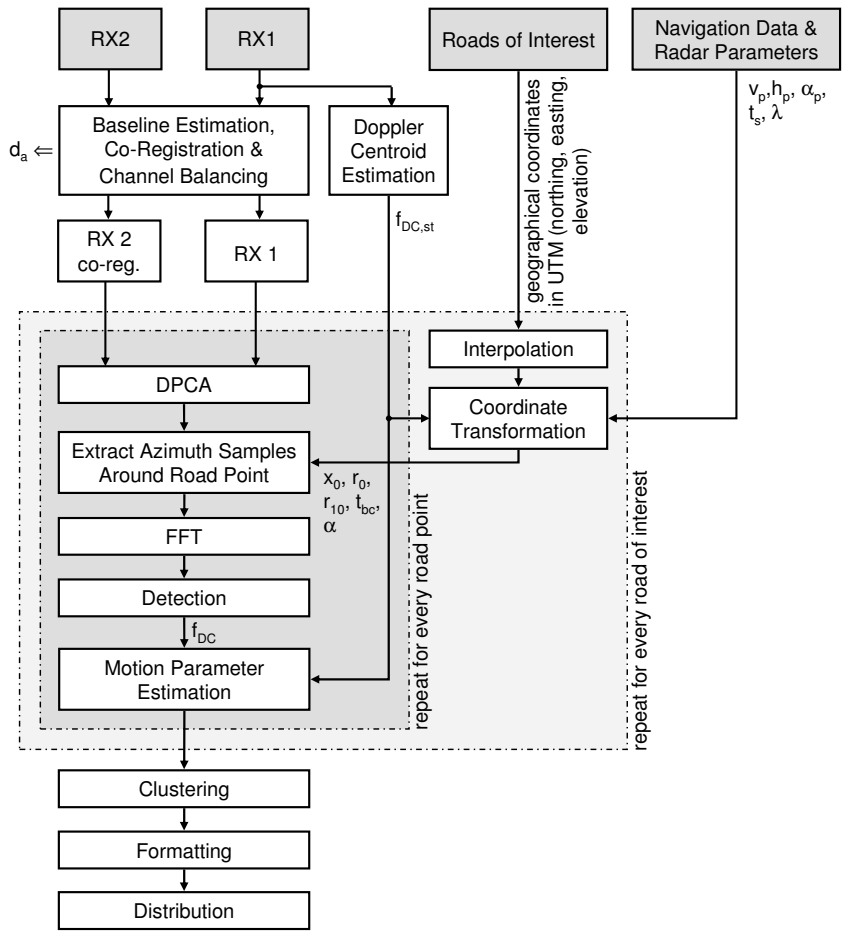

Fig. 3. Simplified flow chart of the proposed algorithm.

data to the traffic management center, a clustering operation is performed, where multiple detections of one and the same vehicle are merged to only one physical vehicle (cf. Fig. 3 bottom). The whole algorithm sketched in Fig. 3 can also be used for single-channel systems by just omitting the stages "Baseline Estimation, ..." and "DPCA". With single-channel systems only fast moving vehicles falling outside the clutter band are detectable, but for these fast vehicles the parameters absolute velocity, heading and geocoded position can be estimated with high accuracy. Furthermore, instead of having only two channels also multiple channels and more sophisticated techniques like space-time adaptive processing [2] can be applied at the cost of increased computational load.

\subsection{Coordinate Transformation}

The relation of the global Cartesian UTM coordinate system $\left\{x_{U T M}, y_{U T M}, z_{U T M}\right\}$ and the local Cartesian coordinate system $\{x, y, z\}$ relevant for GMTI processing is sketched in Fig. 4. The $x$-axis is defined by the platform velocity vector $\vec{v}_{p}$, which is assumed to be constant. A squinted geometry has to be considered, since in general it can not be ensured that the squint angle and, hence, the Doppler centroid of the clutter is negligibly small. In Fig. 5 it is shown how the received non-squinted and squinted data of one and the same stationary road point are stored in the range-compressed SAR data array. In Fig. $5 x_{r 0}$ is the azimuth position of the road point at minimum range $r_{0}, x_{0}$ is the azimuth offset due to 


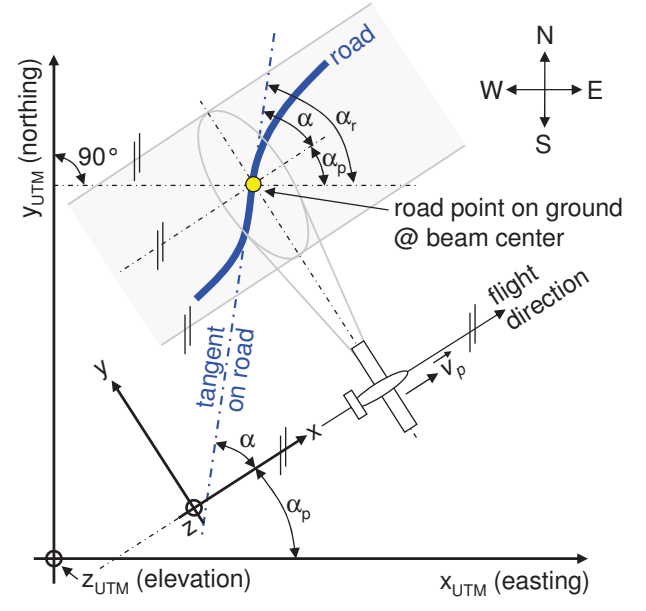

Fig. 4. Relation between the global geographical UTM and the local Cartesian coordinate system.

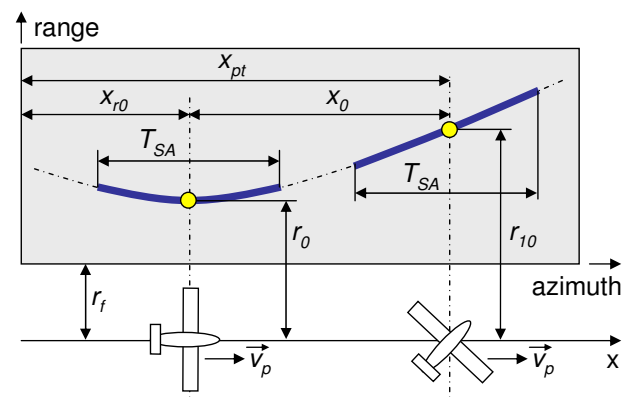

Fig. 5. Range-compressed SAR data array containing a single road point in the non-squinted (left) and squinted (right) case.

the squint angle and $r_{10}$ is the beam center range. By decomposing the range vector $\vec{r}$ into a component parallel and into a component perpendicular to flight direction, the vectors $\vec{x}_{r 0}$ and $\vec{r}_{0}$ are obtained:

$$
\vec{x}_{r 0}\left(t=t_{s}\right)=\left\langle\vec{v}_{p}, \vec{r}\left(t=t_{s}\right)\right\rangle \frac{\vec{v}_{p}}{\left\|\vec{v}_{p}\right\|^{2}},
$$

where $t_{s}$ is the absolute start time of data acquisition, $\langle$.$\rangle is$ the inner product and $\|$.$\| is the \mathrm{L}_{2}$ norm. The vectors $\vec{v}_{p}$ and $\vec{r}$ can be computed using the known UTM coordinates of the stationary road point and the radar platform. The minimum range $r_{0}$ is then given by

$$
r_{0}=\left\|\vec{r}\left(t=t_{s}\right)-\vec{x}_{r 0}\left(t=t_{s}\right)\right\| .
$$

For computing the azimuth position $x_{p t}$ of the road point within the data array the following equation can be used:

$$
x_{p t}=\left\langle\frac{\vec{v}_{p}}{\left\|\vec{v}_{p}\right\|}, \frac{\vec{x}_{r 0}\left(t=t_{s}\right)}{\left\|\vec{x}_{r 0}\left(t=t_{s}\right)\right\|}\right\rangle\left\|\vec{x}_{r 0}\left(t=t_{s}\right)\right\|-x_{0} .
$$

The azimuth offset $x_{0}$ can be computed as

$$
x_{0}=r_{0} \tan \psi,
$$

where the squint angle $\psi$ is given by

$$
\psi=\arcsin \left(\frac{\lambda f_{D C, s t}}{2 v_{p}}\right)=\arccos \left(\frac{r_{0}}{r_{10}}\right) .
$$

In previous equation $\lambda$ is the radar wavelength and $f_{D C, s t}$ the Doppler centroid of the clutter, which can be estimated from the data of a single channel. Knowing the squint angle the beam center range can be computed:

$$
r_{10}=\frac{r_{0}}{\cos \psi}
$$

The beam center time of the road point is given as

$$
t_{b c}=t_{s}+\frac{x_{p t}}{v_{p}}
$$

\subsection{Motion Parameter Estimation}

The motion equations of a vehicle moving at constant altitude $h_{v}$ can be written as

$$
\begin{aligned}
& x_{v}=x_{p t}+v_{0}\left(t-t_{b c}\right) \cos \alpha+\frac{1}{2} a\left(t-t_{b c}\right)^{2} \cos \alpha, \\
& y_{v}=y_{0}+v_{0}\left(t-t_{b c}\right) \sin \alpha+\frac{1}{2} a\left(t-t_{b c}\right)^{2} \sin \alpha,
\end{aligned}
$$

where $v_{0}$ is the absolute velocity at beam center time $t_{b c}, a$ is the constant acceleration and $\alpha$ is the road angle with respect to the $x$-axis (cf. Fig. 4). The across-track position of the target at $t=t_{b c}$ is denoted as $y_{0}$ and given as

$$
y_{0}=\sqrt{r_{0}^{2}-\Delta h^{2}}
$$

where $\Delta h=h_{v}-h_{p}$ is the altitude difference between the moving vehicle and the radar platform. The distance from the transmit antenna to the target is then

$$
r(t)=\sqrt{\left[x_{v}-x_{p t}+x_{0}-v_{p}\left(t-t_{b c}\right)\right]^{2}+y_{v}^{2}+\Delta h^{2}} .
$$

After performing a second order Taylor expansion and some substitutions the range can be approximated as [3]

$$
r(t) \cong r_{10}-\frac{\lambda}{2} f_{D C} \cdot\left(t-t_{b c}\right)-\frac{\lambda}{4} k_{a} \cdot\left(t-t_{b c}\right)^{2}
$$

where $f_{D C}$ is the total Doppler shift of the received signal due to squint and target motion and $k_{a}$ is the Doppler slope. The Doppler shift $f_{D C}$ can be estimated after transforming the azimuth samples around the road intersection point (cf. Fig. 2 right) into Doppler domain. The absolute beam center vehicle velocity can then be computed as

$$
v_{0}=\left|\frac{\lambda r_{10}\left(f_{D C, s t}-f_{D C}\right)}{2\left(x_{0} \cos \alpha+y_{0} \sin \alpha\right)}\right|=\left|v_{a b s}\right| .
$$

The heading of the vehicle is given by

$$
\alpha_{v}=\left\{\begin{array}{rl}
\alpha & \text { if } \operatorname{sgn}\left(v_{a b s}\right)=+1 \\
\alpha-180^{\circ} & \text { if } \operatorname{sgn}\left(v_{a b s}\right)=-1
\end{array},\right.
$$

where $\operatorname{sgn}($.$) is the signum function.$ 


\section{EXPERIMENTAL DATA}

In 2007 several GMTI experiments have been conducted using DLR's new F-SAR system [4]. As test sites an airfield in Memmingen and a region around the Chiemsee, both located in Germany, have been used. F-SAR has been operated in Xband in a dual-channel mode. Some of the controlled vehicles were equipped with GPS to gain reference positions and velocities for the GMTI algorithm verification. Simultaneously with the radar optical images from the same scene were taken. In Fig. 6 the obtained GMTI results from a data take acquired
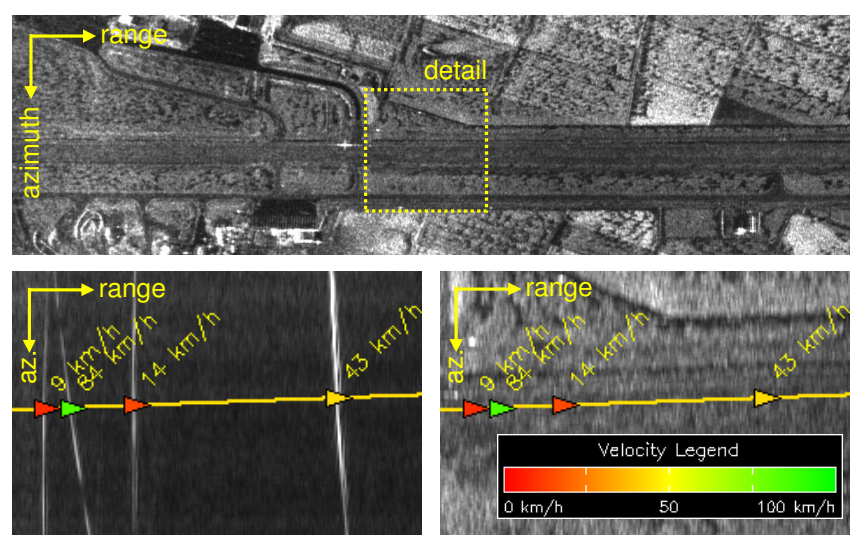

Fig. 6. SAR image of Memmingen airfield (top), rangecompressed DPCA image of the "detail" with overlaid runway axis and detected moving vehicles as triangles (bottom left) and corresponding SAR image (bottom right).

over the Memmingen airfield are shown. All controlled vehicles have moved on the runway in across-track direction. The estimated velocities are $8.6,84.2,14.2$ and $42.7 \mathrm{~km} / \mathrm{h}(128$ azimuth samples were used for parameter estimation). Compared to the reference data the velocity estimation errors are $-1.5,3.5,-1.8$ and $-1.3 \mathrm{~km} / \mathrm{h}$. The position errors are 17.9, 9.9, 17.3 and $16.5 \mathrm{~m}$. The runway in Memmingen is about $30 \mathrm{~m}$ broad and as road axis for the coordinate transformation the middle of the runway was chosen, but during the experiment the vehicles have moved on the edge. Under this aspect, the estimation accuracy is quite good. In the "Formatting" stage also KML files are produced, which easily can be visualized using Google Earth as shown in Fig. 7. Here a GMTI result of a Chiemsee data take, where a lot of customary road vehicles have been detected on the autobahn $\mathrm{A} 8$, is visualized.

\section{CONCLUSIONS}

A GMTI algorithm suitable for single- and multi-channel SAR data based on a priori knowledge was presented. The algorithm was verified using real dual-channel SAR data acquired with DLR's airborne system F-SAR. The obtained performance implies that the algorithm is applicable for realtime traffic monitoring applications.

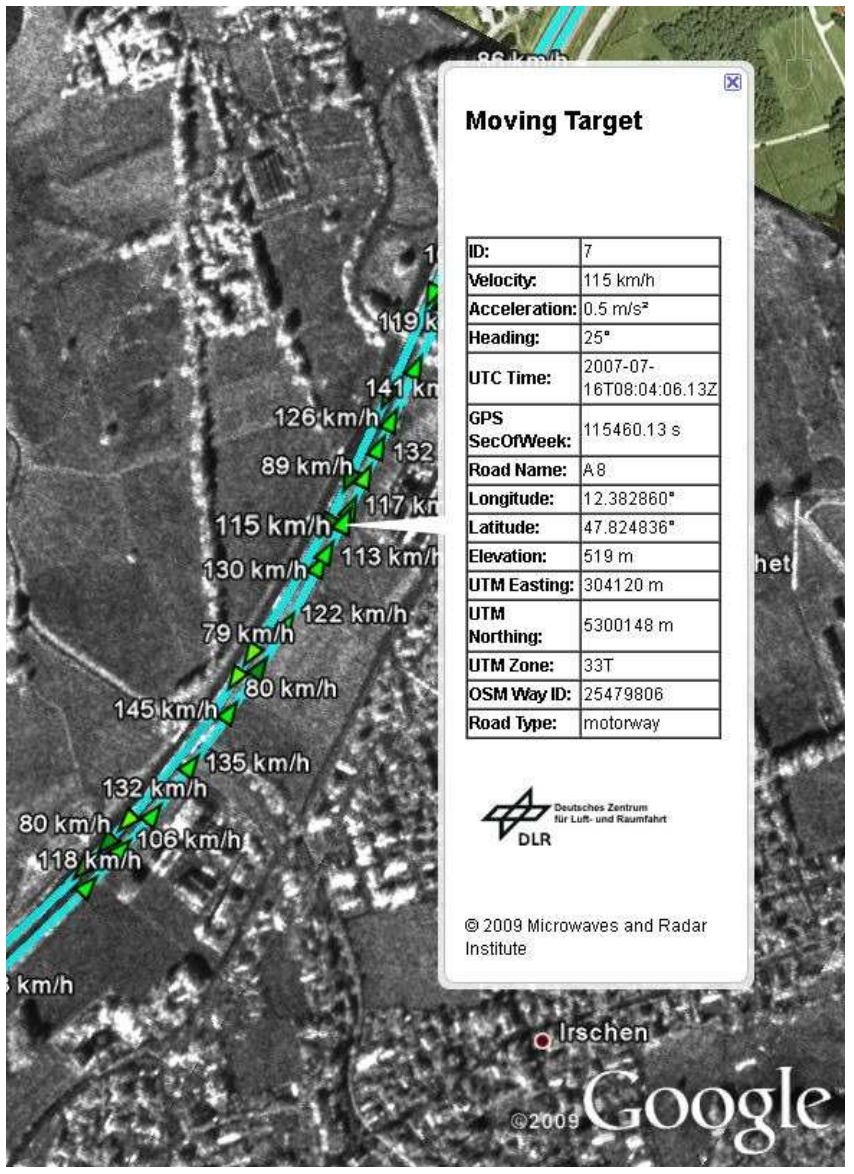

Fig. 7. Quick-look F-SAR image (size $1.5 \times 0.9 \mathrm{~km}$ ) of the autobahn A8 as Google Earth overlay. The shown vehicles were automatically detected and their parameters were automatically estimated using the proposed algorithm.

\section{REFERENCES}

[1] F. Meyer, S. Hinz, A. Laika, D. Weihing, and R. Bamler, "Performance analysis of the TerraSAR-X traffic monitoring concept," ISPRS Journal of Photogrammetry and Remote Sensing, vol. 61, no. 3-4, pp. 225-242, 2006.

[2] R. Klemm, Space-Time Adaptive Processing: Principles and Applications. UK: IEE, 1998.

[3] S. V. Baumgartner and G. Krieger, "SAR Traffic Monitoring Using Time-Frequency Analysis for Detection and Parameter Estimation," in IEEE International Geoscience and Remote Sensing Symposium (IGARSS), vol. 2, Boston, USA, July 2008, pp. II-25 - II-28.

[4] R. Horn, A. Nottensteiner, and R. Scheiber, "F-SAR DLR's advanced airborne SAR system onboard DO228," in 7th European Conference on Synthetic Aperture Radar, vol. 4, Friedrichshafen, Germany, June 2008, pp. 195198. 